\title{
PETRÓLEO BRASILERO S.A.: UNA EMPRESA ESTATAL BRASILEÑA INTERNACIONALIZADA*
}

\author{
Armando Dalla Costa \\ Universidad Federal de Paraná, Brasil \\ ajdcosta@uol.com.br \\ Huáscar Fialho Pessali \\ Universidad Federal de Paraná, Brasil \\ Sandra Cristina Da Cunha Gonçalves \\ Universidad Federal de Paraná, Brasil
}

\section{RESUMEN}

Petróleo Brasileiro S.A. (Petrobras) es la mayor empresa estatal brasileña y tiene una sólida trayectoria de internacionalización. El objetivo de este artículo es entender cómo la empresa creció en el mercado interno y, bajo el amparo de las teorías de internacionalización de la firma, comprender cómo fue su avance en el mercado internacional. Inicialmente, el texto rescata el origen y el desarrollo de la empresa. Luego, verifica cómo comenzó y se expandió su internacionalización para tres grandes áreas geográficas (América, Eurasia y África), en un contexto de disputa global por el petróleo a través de concesiones de explotación, procesos de refinamiento y distribución. Como conclusión previa se puede afirmar que su principal fuente de ingresos continúa siendo el mercado brasileño, pero la inserción en los mercados internacionales ha crecido, ya sea en valores absolutos como en términos de sofisticación de actividades, pasando de una empresa que buscaba insumos para una nueva fase a la venta de productos finales y tecnología de explotación en aguas profundas y ultra profundas.

Palabras clave: Empresas públicas, Petrobras, Internacionalización, petróleo y derivados Brasil.

* Texto traducido del portugués por José María Las Heras Aliciardi, Magíster en Economía por el Programa de Posgrado en Desarrollo Económico de la Universidad Federal de Paraná, Curitiba, Brasil. 


\title{
PETRÓLEO BRASILEIRO S.A.: AN INTERNATIONALIZED BRAZILIAN STATE COMPANY
}

\begin{abstract}
Petrobras is the largest governmental firm in Brazil and has a solid path of internationalization. This paper explores how Petrobras grew in the domestic market and, in the light of the theory of the internationalization of the firm, moved on towards the international market. First, it shows the beginning steps of Petrobras. It then follows Petrobras' internationalization moves to three geographic areas (America, Eurasia and Africa), all in a context of a global dispute for oil exploration, production and distribution. In the conclusion, it is showed that Petrobras' main revenues still come from the Brazilian market. Its expansion to international markets, however, has grown in absolute terms and also in sophistication. Petrobras started with a strategy of searching for new sources of oil (inputs) and progressed to a new phase, focused on selling final goods and developing technology for exploration in deeper waters.
\end{abstract}

Key words: Public companies, Petrobras, Internationalization, petroleum and derivates, Brazil. 


\section{INTRODUCCIÓN}

En septiembre de 2010, Petrobras consiguió US\$ 69.970.000.000 en la mayor venta de acciones jamás realizada en el mercado mundial de capitales. Después del lanzamiento, su valor de mercado alcanzó los US\$ 270.000.000.000, tornándose la segunda mayor petrolera del mundo, detrás de Exxon (US\$ 313.000.000.000) y delante de Petro China (US\$ 266.000.000.000) (Sciarretta 2010).

De acuerdo con Petrobras, estos recursos serán utilizados, fundamentalmente, para viabilizar la explotación de pre-sal ${ }^{7}$. Para esto, el Consejo de Administración aprobó el Plan de Negocios 2012-2016, con una inversión total de US\$ 236.500.000.000 (Petrobras 2011). La meta de Petrobras es llegar en 2014 a los 3.900.000 de barriles de petróleo equivalente por día (BOE) y en 2020 a los 5.400 .000 de BOE.

En 2012, la ganancia neta de Petrobras fue de US\$ 11.100.000.000 y se prevé para 2013 inversiones por el valor de US\$ 51.300.000.000, principalmente en la explotación y producción en Brasil (G1 2013). La empresa cuenta con una estructura industrial compuesta por 109 sondas de perforación, 13.174 pozos productores (gas y petróleo), 125 plataformas, producción diaria de 2.600 .000 barriles de petróleo y 452.000 barriles de gas natural (producción interna y externa), 16 refinerías (en Brasil y en el exterior), 242 barcos (entre flota propia y de terceros), 8.356 puestos de venta, 2 fábricas de fertilizantes, 7 usinas de biocombustibles, 16 usinas termoeléctricas y 4 usinas de energía eólica (Petrobras 2011).

Petrobras contaba, a finales de 2012, con un cuadro de 85.065 empleados, de los cuales 7.640 se desempeñaban en el exterior ${ }^{8}$. Con relación a la facturación de la compañía, la participación internacional en 2012 representó el 12,4\%, lo que significó US\$ 18.400.000.000 sobre los US\$ 148.000.000.000 de las ventas totales ${ }^{9}$.

7 En la explotación de petróleo en el mar, después de la lámina de agua siguen las capas de pos-sal y pre-sal. La capa pre-sal se encuentra a una profundidad de aproximadamente 7.000 metros debajo de la lámina de agua. En 2007, Petrobras descubrió la existencia de petróleo y gas en la capa pre-sal, a 800 kilómetros de distancia del litoral brasileño. La explotación de petróleo en el pre-sal se inició en 2009 y ya a comienzos de 2013 producía 200.000 barriles por día. De acuerdo con el Plan de Negocios de la compañía, hasta el año 2016 deberán ser invertidos US\$ 69.600.000.000 para la explotación en la capa pre-sal, lo que permitirá en el año 2017 alcanzar la producción de 1.000 .000 de barriles por día en aguas ultra profundas (Petrobras 2013).

8 Petrobras. Relatório de Actividades 2012 [05-03-20I3].

9 Petrobras. Relatórios Contables [05-03-2013]. 
Al momento de su fundación, el 3 de octubre de 1953, ni los más optimistas conseguían proyectar una empresa que alcanzara semejante tamaño, que se volvería la más importante transnacional brasileńa y que podría llevar al país a la autosuficiencia en la extracción y refinamiento de petróleo.

Este artículo tiene como objetivo principal examinar la actuación de Petrobras a lo largo de su historia en relación a los mercados internacionales. Para esto, en una primera parte se presentan algunas reflexiones teóricas que ayudan a entender el proceso de internacionalización de las empresas. Luego, se analiza su historia, actuación y crecimiento en Brasil, y su contribución para llevar al país a la autosuficiencia en petróleo y derivados en el inicio del siglo XXI. En una tercera parte, se estudia como se dio el ingreso y desarrollo de las actividades de la empresa en otros países de América, Eurasia y África. Finalmente, presentamos las principales conclusiones.

\section{ELEMENTOS TEÓRICOS PARA ENTENDER LA INTERNACIONALIZACIÓN DE PETROBRAS}

De acuerdo con Alem y Cavalcanti (2007: 260), "a grande complexidade do processo de internacionalizaçáo que envolve empresas e países de estruturas bastante diferenciadas não permite que haja uma teoria geral sobre o processo". A pesar de esto, existen en la literatura varios esfuerzos de interpretar y sistematizar el fenómeno de internacionalización de empresa ${ }^{10}$. Buena parte de la literatura se enfoca en el movimiento de empresas de países más desarrollados hacia países menos desarrollados, un fenómeno característico desde los ańos 1950 (Hymer 1968). Aun mucho menos sistematizadas, existen también discusiones sobre la internacionalización de empresas nativas de países menos desarrollados (Lecraw 1977, Lall 1983), un fenómeno que ganó mayor dimensión a partir en la segunda mitad de los ańos 1970 (UNCTAD 2007).

De acuerdo con el abordaje de Dunning (1988), para que las empresas se internacionalicen, éstas deben poseer ciertos tipos de ventajas sobre sus competidores que justifiquen la inversión directa en el exterior. Las llamadas

io Se destaca en Brasil la Fundación Dom Cabral, centro de desarrollo de ejecutivos, empresarios y empresas, creada en 1976 a partir del Centro de Extensión de la Pontificia Universidad Católica de Minas Gerais. En I993, asociada a un grupo de empresas, crearon el Núcleo de Negocios Internacionales, que intensificó estudios e investigaciones sobre el tema (http://www.domcabral.org.br). Se destaca también el Núcleo de Investigación en Internacionalización de Empresas, relacionado al COPPEAD de la Universidad Federal de Río de Janeiro, que se inició en I 977 con el Grupo de Investigación en Gerencia de Exportación (http://www.nupin.coppead. ufrj.br). 
"ventajas de propiedad", por ejemplo, incluyen aquellas relacionadas a los activos tangibles e intangibles (marcas, capacitación tecnológica, calificación de la mano de obra) que permiten que las firmas puedan aprovechar las ventajas de localización ofrecidas por otros países, como recursos naturales, mano de obra, infraestructura y tamaño de mercado.

Dunning (1988) divide la motivación de las empresas en dirección a la internacionalización en cuatro elementos: 1) búsqueda de recursos naturales y ventajas comparativas; 2) mayor facilidad de comercialización; 3) acceso a nuevos mercados; 4) incremento de la eficiencia. Con relación a la internacionalización que busca el acceso a recursos y utilización de ventajas comparativas, se destaca en la experiencia internacional la búsqueda de materias primas y mano de obra más baratas que en el país de origen. Esto haría más viable la producción destinada a otros mercados con costos más competitivos a nivel internacional. Respecto de la mayor facilidad para la comercialización se encuentra, por ejemplo, la instalación de oficinas de representación ayuda a garantizar la disponibilidad del producto en el mercado que se pretende ingresar al establecer algún control sobre canales locales de distribución. El acceso a nuevos mercados puede combinar varios factores, como la existencia de de barreras comerciales naturales (dificultades físicas) o creadas (restricciones tarifarias). La búsqueda de la eficiencia se vincula con la racionalización de la producción para explotar economías de especialización y localización. En un grado más avanzado de internacionalización, las empresas multinacionales distribuyen etapas de sus actividades productivas en países diferentes que presenten diversas ventajas en la producción de una etapa específica. Lo que originalmente puede ser visto como una filial, deja de ser sólo una fiel "reproducción" de la matriz, pudiendo especializarse en una o algunas etapas específicas de la producción. Casson (1983) y Guilpin (1987) caracterizan esto como una verticalización internacional de la empresa.

Estudiando casos de internacionalización de empresas brasileñas, Iglesias y Motta Veiga (2002) mencionan algunos tipos de inversiones externas que apoyan la expansión de sus exportaciones: oficinas comerciales, almacenes, centros de distribución, montaje o terminación del producto en el mercado de destino o para reexportación, asistencia/servicio post venta, laboratorio y talleres para adaptación de productos con vista en el mercado importador. A estos, los autores adicionan el establecimiento de comercios, puntos de venta propios y las franquicias. En estas modalidades, el emprendimiento en otros países busca mejorar o tornar viable la atención directa a los clientes internacionales de productos originarios de la matriz de la empresa. 
La amplitud y diversidad de las inversiones, como las mencionadas arriba, es típico de empresas con mayor experiencia y conocimiento del mercado internacional y, generalmente, incluyen acuerdos comerciales con socios locales. Tales acuerdos, por lo general, implican un incremento en el número de estos convenios estratégicos en Brasil direccionados a los negocios de la empresa con el exterior, y el surgimiento de grupos de socios extranjeros. De esta manera, a los desafíos de la naturaleza productiva del mercado se suman los relacionados a la gestión internacional. En este contexto, Chandler $(1962,1977)$ ayuda a explicar cómo las empresas tratan de superar estos obstáculos a través de innovaciones organizacionales que envuelven principalmente la descentralización de decisiones y el aprovechamiento las ventajas locales. Como observan Buckley y Casson (1976) y Caves (1980), la internacionalización mediante la inversión extranjera directa sería semejante a los procesos de integración y diversificación de la firma en mercados geográficamente diversos dentro de un mismo país, tanto en las motivaciones como en las maneras organizacionales de viabilizarlos. A esto, sin embargo, se suman los costos de transacción necesarios para compatibilizar en algún grado la realización de negocios en ambientes institucionales distintos.

Otro aspecto teórico que ayuda a entender la experiencia de internacionalización de Petrobras es la búsqueda de respuestas para los siguientes asuntos: ¿Por qué las firmas deciden entrar al mercado internacional? ¿Qué y cuáles son los tipos de activos y/o actividades transferidas para el mercado internacional? ¿Cuándo es el momento adecuado para internacionalizarse? ¿Dónde es relevante tener actividades en el exterior? ¿Cómo o qué modelo utilizar para insertarse en el mercado internacional?

En este contexto, las corrientes teóricas se dividen, a grandes rasgos, en: teorías con bases económicas tradicionales (normativas) y con bases económicas comportamentales (descriptivas).

La primera corriente busca analizar la internacionalización de las firmas como consecuencia de tomada de decisiones racionales, que persiguen la optimización de beneficios por medio del crecimiento en otros mercados. La segunda entiende que la internacionalización depende de actitudes, percepciones y comportamientos de los agentes (firmas y gerencias), que se relacionan a la reducción de los riesgos y costos de transacción en las decisiones sobre dónde y cómo expandir (Carneiro et al. 2005; Pitelis y Sugden 2000).

Relacionado a las inversiones directas en el extranjero (IDE), Johanson y Wiedersheim-Paul (1999), citan que muchas firmas pasan por un 
proceso gradual de sucesivo aumento en el comprometimiento con su internacionalización. Un ejemplo del desarrollo de éste: 1) no existe en principio la actividad exportadora; 2) la exportación comienza a realizarse por medio de representantes internacionales independientes; 3) las ventas ocurren a través de subsidiarias propias de la firma; y 4) se inicia la producción en plantas instaladas en el exterior. Ésta es una interpretación clásica que se conoce como modelo Uppsala, la universidad sueca que fue promotora de su desarrollo.

Dicho modelo sufrió diversas críticas y revisiones, por más que todavía ayude a explicar muchos casos y sirva de punto de partida para otros análisis. Los casos más recientes de fusiones y adquisiciones, por ejemplo, son de empresas que trascienden al abordaje gradual descripto en el modelo de Uppsala y muestran la dinámica de la internacionalización de los capitales productivos. Estudios de caso apuntan para diversas ocasiones y modalidades en que las empresas "quemaron" niveles en su internacionalización, principalmente las oriundas de países emergentes (Kuada y Sørensen 2000). Cantwell y Narula (2003), por ejemplo, evidencian casos en que las empresas proceden directamente a la inversión directa extranjera sin haber sido antes exportadoras. Varias de ellas aprovechan oportunidades tecnológicas o institucionales en que el primer movimiento permite alguna ventaja de monopolio por tiempo suficiente para el aprendizaje y adaptación al nuevo ambiente de negocios en el país receptor.

Dunning $(1981,2001)$ observa que determinadas empresas de los países emergentes, en general, cuentan con alguna especificidad local que resulta impregnada en la trayectoria de internacionalización, dado que otros factores como escala y dominio de conocimientos complejos favorecen a empresas de países desarrollados. Lall (1983) argumenta que las empresas de países emergentes adaptan o desarrollan tecnologías oriundas de los países desarrollados para utilizarlas en sus mercados específicos, lo que llamó de "cambio tecnológico localizado". Como resaltan Carvalho y Goldstein (2008), esto contradice la idea de que tales empresas apenas aprovechan recursos locales pre existentes para capitalizar ventajas comparativas. Ellas también desarrollan competencias tecnológicas que pueden eventualmente ser aprovechadas para la internacionalización. Esto se potencia con el crecimiento del ingreso del país de origen y el respectivo crecimiento de escala de operación de la empresa.

Carvalho y Goldstein (2008) destacan el caso específico de la industria del petróleo y mencionan su creciente oligopolización en escala global. Esto se dio en función tanto de la escala de operaciones como del alto 
riesgo de las inversiones, como de la inseguridad en el acceso a las reservas. Así, muchos gobiernos de países emergentes constituyeron empresas estatales como forma de compensar las distorsiones del oligopolio de empresas privadas de países desarrollados y reducir la dependencia para con los pocos y poderosos abastecedores. La UNCTAD (2007) registra, por ejemplo, que 34 de las 50 mayores empresas del sector son estatales. Varios países emergentes trataron de negociar el acceso a sus reservas con contrapartidas de acceso a la tecnología detentada por las empresas de los países desarrollados (estrategia que continúa siendo utilizada) con el objetivo de acelerar el esfuerzo del catching up tecnológico de sus empresas. Y, como sugiere Goldstein (2009), al dominar el conocimiento transmitido y utilizarlo en combinación con las especificidades requeridas por los mercados locales, algunas de las empresas de países emergentes consiguieron desarrollar competencias suficientes para aprovechar o crear oportunidades de participación en los mercados internacionales

\section{PETROBRAS Y EL INICIO DE LA EXPLOTACIÓN DE PETRÓLEO EN BRASIL}

Existen registros que indican el inicio de la explotación de petróleo para fines comerciales que datan de la década de 1820 en West Virginia (McKain y Allen 1994), más allá de que sea común asignar a Edwin Drake la perforación del primer pozo de petróleo en 1859 en Pensilvania. De todos modos, el pozo de Drake tenía 22 metros de profundidad y producía inicialmente 25 barriles por día (Moura 2003: 96).

En Brasil, los primeros sondeos profundos fueron realizados durante la década de 1890 en el estado de San Pablo. Sin embargo, las primeras reservas petrolíferas en territorio nacional fueron recién descubiertas el 21 de enero de 1939, o sea, más de un siglo después de los pioneros trabajos de sondeo realizados en San Pablo. Este descubrimiento resultó en la perforación de 17 pozos en Lobato, estado de Bahía, por técnicos del Departamento Nacional de Producción Mineral, aún con carácter experimental. No obstante, fue sólo en mayo de 1941 que se produjo el descubrimiento en Candeias (BA) del primer campo comercial de petróleo del país. En diciembre de 1942, la producción brasileña de petróleo ya alcanzaba los 32.000 barriles, y un ańo después llegaría a los 48.151 (Moura 2003).

Con la finalización de la Segunda Guerra Mundial, se planteó en el escenario internacional una fuerte disputa, ya librada en años anteriores, entre defensores del ingreso de empresas extranjeras para explotar el petróleo y nacionalistas, contrarios a la presencia de multinacionales en el sector. La contienda tomó fama por la frase: O petróleo é nosso, utilizada 
en las campañas para la defensa de la creación de una empresa estatal encargada de la prospección, explotación y refinamiento.

El resultado de la controversia fue la creación en 1938, por obra del presidente Getúlio Vargas, del Consejo Nacional del Petróleo (CNP), acto que aumentaría el control estatal sobre las actividades del sector petrolífero. Más tarde, el presidente Vargas daría sanción el 3 de octubre de 1953 a la Ley N. ${ }^{\circ}$ 2.004/1953, creando, así, a la empresa Petrobas ${ }^{11}$.

El gobierno federal participaba mayoritariamente en la empresa y, de esa forma, ejercía control pleno sobre las actividades de explotación y procesamiento. Vale la pena resaltar que Petrobras y sus subsidiarias tenían el monopolio de tales actividades, apenas la distribución era la excepción (Moura 2003: 56). El monopolio de explotación y producción prevaleció hasta 1997, cuando fue publicada la Ley N.o 9.478/1997, alterando la estructura de mercado.

Por lo tanto, desde el inicio Petrobras estaría encargada tanto de la prospección como de la explotación y del refinamiento, debido a que detentaba el monopolio de esas actividades. Como consecuencia de esa estrategia gubernamental, la empresa absorbió del CNP una refinería en funcionamiento y otra en construcción. Además de esto, habían otras cinco pequeñas usinas privadas en actividad o en construcción: la Destilería RioGrandense de Petróleo, la Ipiranga (funcionando desde los ańos 1930), Capuava y Manguinhos (inauguradas en 1954) y Manaus (inaugurada en 1956). Sin embargo, las primeras refinerías construidas por Petrobras sólo serían inauguradas en la década de 1950. Están entre ellas la refinería Presidente Bernardes, en 1956, instalada en la ciudad de Cubatão, San Pablo, y Refinería Duque de Caxias, en la Baixada Fluminense en 1961, resultando que por la primera vez la capacidad de refinación en el país superara el consumo (Moura 2003: 120-123).

Otros aspectos asociados a la estrategia de negocios de la empresa eran las inversiones realizadas en investigación y desarrollo de nuevas tecnologías, táctica que marcaría una trayectoria. El inicio de este proceso se dio en 1957 cuando fue creado el Centro de Aperfeiçoamento e Pesquisas de Petróleo (Cenap). El Cenap fue reestructurado en 1963, tornándose el Centro de Pesquisas e Desenvolvimento (Cenpes), que pasó a contar con los 67 empleados de la antigua institución. Petrobras invertiría hasta aquel entonces US\$ 80.000 en investigación, US\$ 10.000 .000 en unidades piloto y cerca de US\$ 110.000 en equipamientos de laboratorio (Moura 2003: 126). La estructura del Cenpes estuvo dispersa hasta 1973 cuando

I I Para más detalles sobre la historia de la empresa véanse, entre otros, a Moura (2003), y Alveal (2008) para un análisis crítico sobre la creación y desarrollo de la empresa. 
sus unidades fueron centralizadas en un terreno de la Universidade Federal do Rio de Janeiro (UFRJ). En esta estructura tuvieron lugar las primeras investigaciones offshore, las cuales se mostraron esenciales para el mantenimiento de la empresa en el sector y, también, en su internacionalización.

La ampliación y la regionalización de la capacidad de producción de la empresa fueron aseguradas con la construcción de las refinerías de Gabriel Passos en Betim (Minas Gerais) y la de Paulinia (San Pablo), inauguradas en 1968. Paralelamente a estas construcciones, en el inicio de la década de 1970 fueron expropiadas las cuatro refinerías privadas en operaciones en el país. Dos de ellas fueron incorporadas al patrimonio de Petrobras: la refinería de Manaus, en 1972 y la de União, en 1974. Las otras dos fueron desactivadas en 1974: la destilería Rio Grandense (RS) y la refinería de las Industrias Matarazzo de Energía en San Pablo (Moura 2003: 147).

En diciembre de 1967 se creó Petrobras Química S.A. (Petroquisa), la que construyó el polo petroquímico de San Pablo. En 1973 se constituyó otra subsidiaria, Petrobras Fertilizantes S.A. (Petrofértil), la cual absorbió el antiguo Conjunto Petroquímico de Bahía. Se formó así el holding Petrobras, manteniendo el monopolio en el área de prospección, explotación y refinamiento de petróleo, teniendo subsidiarias (Braspetro, Petroquisa y Petrofértil) actuando en áreas específicas.

Frente a las limitaciones técnicas y estructurales de prospección y refinamiento de petróleo, acompañadas de intereses del gobierno brasileño de intensificar la industrialización de la economía, Petrobras se mostró un importante instrumento impulsor de este proceso. En este sentido, la creación de las subsidiarias y la construcción de las refinerías representan la materialización de esa importancia para la industrialización brasileña. Se observa, por lo tanto, que importantes avances estructurales y estratégicos a lo largo de la historia de la empresa estaban, y todavía están, estrictamente asociados a los intereses nacionales.

La primera crisis del petróleo, iniciada en 1973, alteró profundamente la dinámica del sector petrolífero nacional. El abrupto incremento del precio del barril de petróleo estimuló, principalmente en los países importadores de ese producto, la inversión en fuentes alternativas de combustibles. En el caso brasileño, la decisión fue invertir en la producción y uso de biocombustibles, lo que resultó en el lanzamiento del Programa Nacional do Álcool (Proálcool), presentado oficialmente por el Gobierno en 1975.

Un segundo conjunto de acciones fue realizar inversiones para intensificar el descubrimiento de nuevos pozos de petróleo. En este sentido, 
el hallazgo del campo de Garoupa, en el litoral de Rio de Janeiro, en noviembre de 1974, abrió una nueva y prometedora fase en la explotación y producción de petróleo en Brasil. Los trabajos exigieron el desarrollo de nuevas tecnologías en función de las condiciones específicas de los lugares prospectados, a decenas de kilómetros del litoral y en aguas de grandes profundidades (Gráfico 1).

\section{Gráfico 1: Evolución de la extracción de petróleo onshore, offshore y de la Bahía de Campos (en barriles por día), 1954 - 2008}

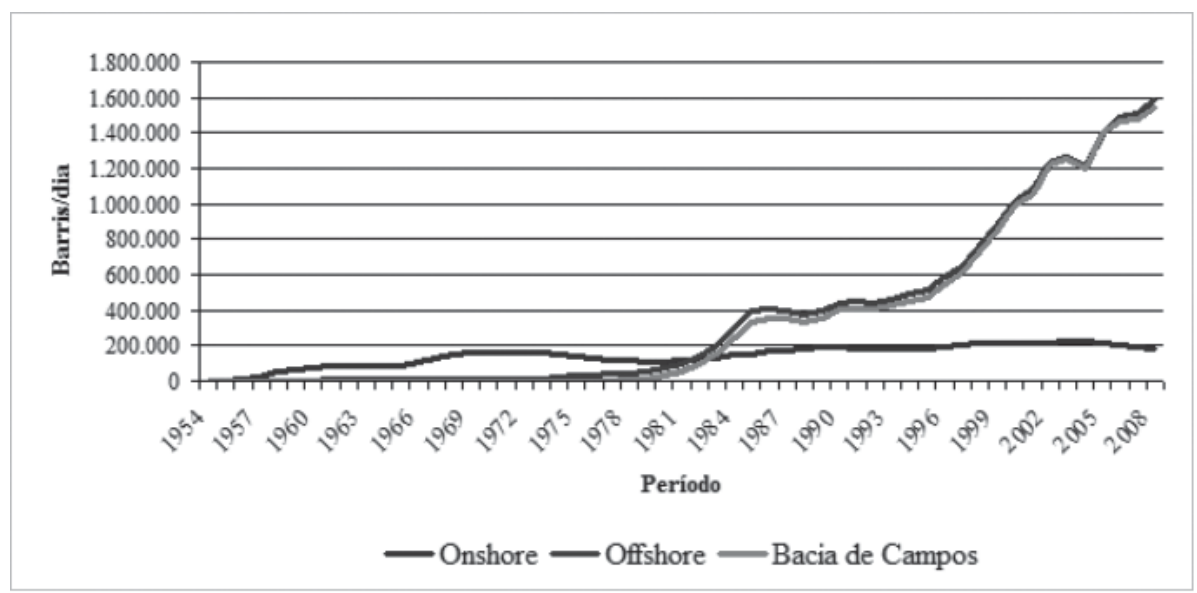

Fuente: Elaborado por los autores, con base en Petrobras (2009b).

Nota: En la explotación offshore se incluye la Bahía de Campos, que entró en operaciones en 1977.

Durante la década de 1980, Petrobras tuvo significativos avances tanto en la explotación de petróleo en alta mar como en el refinamiento y en la distribución. Como vector de comparación, podemos analizar los gastos de importación brasileña de óleo y derivados que en 1981 alcanzaban los US\$ 10.000.000.000 y en 1989 habían sido reducidos para US\$3.000.000.000 (Gráfico 2). Como indicador de los avances de explotación en alta mar, en 1986 Petrobras perforaba pozos en láminas de agua de 1.200 metros de profundidad y extraía petróleo a profundidades en torno de 400 metros, un récord mundial. En 1988 ese récord fue superado por la producción de petróleo en el campo de Marimba, bahía de Campos (RJ), a 492 metros debajo del nivel del mar.

Conforme declaración de Décio Costa (2003), Gerente Ejecutivo de Petrobras para el Cono Sur: 
[...] hoje a produção da Petrobras é, basicamente, fruto das descobertas de águas profundas na Bacia de Campos (RJ), que aconteceram de 1985 em diante. Naquele tempo, quem tinha plataformas de perfuração de petróleo no mar para 800 a 900 metros eram apenas companhias estrangeiras. Acho que no mundo havia 10 ou 12 plataformas dessas e teve uma época que quase todas estavam aqui no Brasil.

\section{Gráfico 2: Evolución del volumen importado, producido y del consumo aparente de petróleo $\left(\mathrm{en} \mathrm{mil} \mathrm{m}^{3}\right), 1955-2007$}

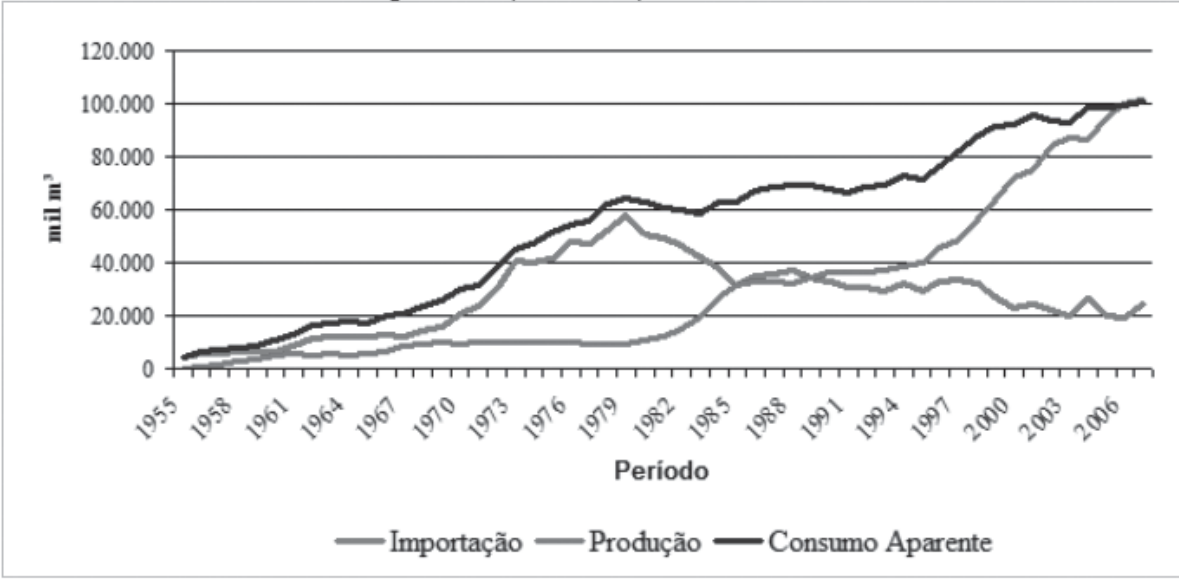

Fuente: Elaborado por los autores con base en Ipeadata (2009).

La década de 1990 representó una transformación en la historia de la empresa, con la extinción del monopolio y la privatización de diversas empresas ligadas directamente con Petrobras. La finalización del monopolio fue resultado de la sanción de la Ley N.o 9.478 el día 6 de agosto de 1997, manteniéndose el monopolio apenas sobre los depósitos de petróleo, gas natural y otros hidrocarbonatos fluidos. Otro hecho que también influenció profundamente la gestión de la firma fue el cambio en la estructura accionaria del holding, producto de las privatizaciones. Las privatizaciones alteraron el control accionario de la empresa en dos tercios, las cuales pasaron para manos de capitales privados, llevando a la empresa a negociar el $40 \%$ de las acciones en la bolsa de Nueva York, pero el gobierno brasileño continuó manteniendo el 56\% del capital votante.

El proceso privatizador se inició durante el primer gobierno electo posterior al período militar (Fernando Collor de Melo, 1990-1992), el cual instituyó el Programa Nacional de Desestatización. El programa afectó directamente las actividades de Petrobras, con la privatización de Petrobras Comercio Internacional (Interbras), de Petrobras Mineración (Petromisa) 
y con la extinción del Consejo Nacional del Petróleo. Poco tiempo después el sector petroquímico de la firma se vio afectado con la venta de Petroflex, Fosfértil, Copesul y la Compañía Álcalis de Rio Grande del Norte.

No obstante, la desestatización no paró. Durante el primer gobierno de Fernando Henrique Cardoso (1995-1999) se produjeron los principales cambios recientes en Petrobras. El 6 de agosto de 1997 se aprobó la "Ley del petróleo", Ley N. 9 9.478, la cual abrió el mercado brasileño al ingreso de otras empresas, es decir, terminó con el monopolio estatal. La Ley limitaba la participación del Estado nacional en el capital de Petrobras al $50 \%$ más una de las acciones con derecho a voto de la compañía, lo que en la práctica forzaba la venta de parte de ese capital en el corto plazo; esto significó prácticamente la privatización de la empresa. A partir de ese momento, Petrobras no podría ser administrada únicamente según los intereses nacionales debido a que debería incorporar en sus decisiones los intereses privados, algo que si bien ya ocurría con anterioridad ahora se tornaría más explícito. Esta situación afectó de manera positiva su internacionalización.

Los cambios de cierta forma no parecen haber interferido significativamente en la continuidad de las inversiones. En 1999, fue elaborado el Plan Estratégico del Sistema Petrobras, estableciendo cinco grandes áreas de negocio: explotación y producción, abastecimiento (incluyendo refinamiento, transporte y comercialización), distribución, energía y gas natural, y petroquímica.

La entrada en operaciones de la Agencia Nacional del Petróleo, Gas Natural y Biocombustibles (ANP) en 1999, trajo el establecimiento del sistema de licitaciones para definir qué empresas tendrían la concesión para la explotación de bloques (o áreas predeterminadas) offshore. En 1998, antes del inicio de las licitaciones, el portafolio de explotación de Petrobras fue estructurado en la forma de concesiones (lo que se conoció como round zero de las licitaciones de concesión), registrándose el derecho de explotación en 397 bloques (ANP 2007). Desde entonces Petrobras participa activamente en las licitaciones de bloques de explotación, muchas veces en conjunto con empresas extranjeras. A fines de 2001, la empresa tenía en operaciones casi 10.000 pozos productores, 31 sondas de perforación y 96 plataformas de producción, alcanzando en promedio una extracción de 1.535 barriles por día (Moura 2003).

En la década de 2000, se torna más explícita la idea de Petrobras de volverse una empresa integrada de energía. Además de la expansión de las actividades petrolíferas, la estrategia de la firma también se orientó para la explotación de gas y, selectivamente, de fuentes renovables de energía, 
destacándose el biodiesel (Petrobras 2004). Al parecer, esta concepción de la no dependencia de una única fuente no renovable y la consecuente búsqueda de nuevas formas de producción de energía capaces de atender las demandas de sustentabilidad ambiental se consolidarán en las estrategias de Petrobras para el futuro próximo.

\section{PETROBRAS INICIA SU INTERNACIONALIZACIÓN CON BRASPETRO}

Se puede decir que la internacionalización de la empresa fue impulsada por dos eventos de gran relevancia que sucedieron durante la década de 1960. El primero, luego de varios intentos frustrados en la búsqueda de petróleo en suelo firme, fue el comienzo de las perforaciones en el mar, en áreas próximas al estado de Sergipe y de Pará. Sin embargo, esta estrategia exigió la importación de equipamientos y la contratación de empresas extranjeras con know-how de explotación marítima.

El segundo evento relevante sucedió en 1963, cuando el gobierno federal atribuyó a Petrobras el monopolio para la importación de petróleo. Estos dos eventos contribuyeron profundamente para que la firma tuviera que operar internacionalmente a través de acuerdos tecnológicos y comerciales con otras compañías, desarrollando mayor conocimiento en la dinámica internacional del petróleo.

A comienzos de la década de 1970, Brasil aún se encontraba inmerso en el período del "milagro económico", caracterizado por la fuerte expansión de las actividades industriales y, en consecuencia, presentando elevadas tasas de crecimiento. Esto, obviamente, exigió mayor dinamismo de la industria petrolera. El crecimiento de la demanda de petróleo incentivó aún más la expansión de Petrobras, lo que incluyó la búsqueda de petróleo en otros países e inversiones en prospección de nuevas reservas.

Para atender el aumento significativo de las operaciones internacionales (comercialización) se creó en 1972 la subsidiaria Petrobras Internacional S.A. (Braspetro). Al poco tiempo de este evento se produjo la primera gran crisis del petróleo, cuando la Organización de los Países Exportadores de Petróleo (OPEP) redujo drásticamente la oferta del producto, resultando en un abrupto incremento del precio en el mercado internacional. La respuesta brasileña a esta situación se dio en varios frentes. Uno de ellos fue la expansión de la oferta interna del producto a través del quiebre del monopolio de explotación y la firma de contratos de riesgo con empresas extranjeras. Otra respuesta vino de la producción de un combustible alternativo, el alcohol. Una tercera fue desarrollar actividades de prospección y explotación de petróleo en campos internacionales a través de Petrobras. 
De acuerdo a lo que fue presentado en la parte teórica del trabajo, la literatura que aborda la internacionalización de las empresas sugiere que uno de los motivos que llevan a las firmas a internacionalizarse es la "búsqueda de recursos naturales" (Dunning 1988). En 1972, a través de la creación de joint ventures con otras empresas, tuvieron inicio las investigaciones de prospección en Colombia, Irak y Madagascar. En este último caso hubo una asociación con Chevron. Al año siguiente, las explotaciones se extendieron a Egipto e Irán, con la participación de la Mobil Oil. Se observa, por lo tanto, que Petrobras expandió su relación con el mercado internacional por medio de la inversión extranjera directa, además de la relación comercial mantenida desde la década de 1960 .

En la segunda mitad de los años 1980 Petrobras buscó reducir sus gastos con suministros del país. En este sentido, como afirma Moura (2003: 198), la empresa intensificó relaciones comerciales con:

Iraque, Irãn, China, Nigéria, Angola, Argélia, Venezuela e Equador, que contribuíram para a reduçáo de $43,6 \%$ nos gastos com compras no exterior em 1986, comparativos ao ano anterior. Quanto às exportações de derivados (sobretudo para os Estados Unidos da América) evoluíram de US\$ 673 milhões em 1985 para US\$ 923 milhóes em 1989, sendo que a gasolina representava 56,3\% desta receita.

Con relación a los trabajos de explotación y producción, Petrobras se instaló en diferentes continentes. En Medio Oriente y Asia fue para Yemen y China; en África se radicó en Argelia y Angola; en América del Norte buscó las reservas del golfo de México; en América del Sur inició actividades en Colombia en 1989, y firmó contratos de explotación y producción offshore en Argentina. En Europa occidental buscó las reservas del mar de Noruega (Moura 2003: 199).

Resulta oportuno destacar un aspecto que se mostró decisivo para impulsar su actuación internacional: el desarrollo de tecnología en investigación, explotación y extracción de petróleo en aguas profundas. Esta tecnología permitió el descubrimiento de reservas de petróleo en la capa conocida como pre-sal y, además de esto, permitió iniciar la explotación de esas reservas.

A partir de mediados de la década de 1970, el Cenpes ya había hecho buena parte del catching up tecnológico con relación a las empresas internacionales más avanzadas y los resultados dieron frutos a lo largo de los años 1980 en la expansión de la producción en aguas profundas. El reconocimiento externo de este esfuerzo vino a través de premios conquistados como el 
Distinguished Achievement de la Offshore Technology Conference, recibido por primera vez en 1992 y que volvería a recibir en 2001. Pero fue a lo largo de la década de 1990 que Petrobras se lanzó al desafío de desarrollar tecnología para la producción en aguas ultra-profundas. Así, a final de los años 1980 el desafío era explotar petróleo a 500 metros de profundidad; en 1999 Petrobras marcó el récord de explotación a 1.853 metros de profundidad en el Campo de Roncador (RJ). En los años 2000, la empresa pasó a explotar reservas que se encontraban en profundidades superiores a 3.000 metros y en alta mar. La tecnología creada y utilizada por la empresa, con acuerdos comerciales nacionales e internacionales, le permitió pasar de importadora para exportadora de productos y proyectos de gran intensidad tecnológica.

\section{PETROBRAS INTERNACIONAL EN EL CAMBIO DE MILENIO Y PERSPECTIVAS}

De acuerdo con informaciones publicadas en el informe anual de Petrobras correspondiente a 2008 (Petrobras 2008), su actuación en el exterior abarca toda la cadena de operaciones de la industria de petróleo y energía, o sea, desde la explotación, producción, transporte, refinamiento y procesamiento de petróleo y gas natural hasta la producción de productos petroquímicos, distribución y comercialización de derivados, y hasta la generación, distribución y transmisión de energía eléctrica.

Para 2008, en el segmento de explotación y producción de petróleo y gas, la empresa tenía activos en 19 países. En el sector de refinamiento actúa directamente en Argentina, Estados Unidos y Japón. En el sector de distribución actúa en Uruguay, Paraguay, Colombia, Argentina y Chile. Vale destacar que en Argentina Petrobras actúa en sectores de petroquímica y energía eléctrica. Mantiene acuerdos de cooperación con Cuba en diversos segmentos de la cadena productiva de petróleo; además de eso, posee oficinas de representación en el Reino Unido, Japón, China y Singapur (Petrobras 2008).

Toda esta actividad está bajo responsabilidad del área internacional de la empresa, una de las directrices del área de negocios creada en el año 2000. El Área Internacional incorporó, así, parte de las actividades de Braspetro, que fue completamente absorbida por Petrobras en 2002 (Petrobras 2007a). Para las operaciones internacionales de la empresa están previstas inversiones de US\$ 11.000 .000 .000 hasta 2016 , de las cuales $80 \%$ deben concentrarse en explotación y prospección (Petrobras 2012).

El inicio de las inversiones directas de Petrobras en el exterior se dio en las Américas, después se expandió para Asia y África. A continuación, 
trataremos de sistematizar una parte de esta trayectoria.

\section{Petrobras Internacional en las Américas}

Colombia fue el país en donde Petrobras inició efectivamente su actuación directa en operaciones internacionales. El ingreso ocurrió en 1972, cuando Braspetro compró concesiones de explotación de la empresa Tennecol (Petrobras 2007a). Al poco tiempo, sin embargo, Petrobras salió del país para regresar recién en 1986 con actividades de exploración. En 2004, se unió con Exxon y la estatal Empresa Colombiana de Petróleo (Ecopetrol), para explorar el área colombiana del Mar del Caribe en aguas de hasta 3.000 metros de profundidad.

De cierta forma, esa cronología representa algunas de las principales acciones adoptadas por la empresa en su internacionalización, no obstante vale la pena algunos otros ejemplos de esta expansión en el continente americano.

Un momento importante fue la entrada en los Estados Unidos en 1987. El ingreso fue resultado de la creación de la subsidiaria Petrobras América Inc., iniciada para la actuación en el Golfo de México y que posteriormente fue ampliada para otras regiones norte americanas.

Paralelamente, fue creada Petrobras Norge, la cual buscaba desarrollar operaciones en el Mar de Noruega, y Brasnor Neptun, una asociación entre Braspetro y Neptun Teknologi, empresa especialista en servicios en aguas profundas (Moura 2003: 199). Todo esto permitió que en 2004 Petrobras consiguiera la concesión para explorar 37 bloques en aguas profundas del Golfo de México, en una región frontal al estado de Texas.

Otro momento destacable de su internacionalización en el continente americano fue su ingreso en Argentina en el año 1993. El proceso se inició con la obtención de concesiones para la exploración y mediante adquisiciones de otras empresas actuantes en ese mercado. La estrategia adoptada para ingresar al mercado argentino la convirtió en una empresa integrada de energía y en una de las mayores productoras de petróleo y gas natural de aquel país. Sus actividades abarcan un conjunto de activos y negocios en las áreas de exploración y producción de petróleo y gas natural, comercialización, refinamiento y procesamiento, distribución de derivados, redes de conductos, petroquímica, generación, distribución y transmisión de energía eléctrica (Petrobras 2007a).

La gestión de negocios en la Argentina se encuentra centralizada en Petrobras Energía S.A., empresa constituida en 2005, como resultado de la 
fusión de Petrobras Argentina S.A. con otras dos firmas de ese país. Cada año se invierten US\$ 500.000.000 en Argentina conforme lo establecido en el Plan de Negocios de Petrobras (Folha de S.Paulo 2012). La producción y reservas de petróleo y gas natural en territorio argentino son las mayores de Petrobras fuera de Brasil. Las demás actividades también se tornaron relevantes en la estrategia de la empresa. En la distribución de combustibles de ese país, por ejemplo, la empresa cuenta con una red de 746 puestos de servicio, generando un total de 5.100 puestos de trabajo para argentinos, brasileños y personas de otras nacionalidades (Petrobras 2007a).

Aun en términos de la inserción en el mercado de América Latina, se destaca la instalación de Petrobras en Bolivia, en el año 1996, concentrándose en la explotación de gas natural. En 1997, la empresa inició la construcción del gasoducto Bolivia-Brasil, obra que tuvo inversiones muy significativas. Paralelamente, la empresa también ingresó en el procesamiento de gas y en el refinamiento de petróleo. En 2001, inició la distribución propia de combustibles en Bolivia, contando con 62 puestos Petrobras. Con relación a la fuerza de trabajo envuelta, en 2006 la firma empleaba 1.533 personas, de las cuales $95 \%$ eran bolivianos. Pasada una década, las inversiones en Bolivia alcanzaron los US\$ 1.000 .000 .000 y, en 2005, sus operaciones representaron el $18 \%$ del PIB boliviano y el $24 \%$ de la recaudación total de impuestos (Petrobras 2007a).

$\mathrm{Su}$ presencia en Bolivia siguió de acuerdo a lo planeado hasta la nacionalización de las refinerías el primero de mayo de 2006, por el presidente Evo Morales. Después de largas negociaciones, el 28 de octubre de 2006 se firmó un acuerdo entre Petrobras y la empresa Yacimientos Petrolíferos Fiscales Bolivianos, especificando los nuevos límites de actuación de cada una en Bolivia. Como consecuencia de esta acción, Petrobras se encuentra actualmente redimensionando sus inversiones en ese país. De acuerdo con manifestaciones de especialistas, se produciría una nueva orientación de los recursos para proyectos en Brasil relacionados al gas natural, por más que no se espere que la empresa necesariamente disminuya su actuación en ese país.

En 2009, Petrobras pasó a operar en Chile a través de la adquisición de 230 puestos de combustibles de la empresa Esso Chile Petrolera por US\$ 500.000.000. Con esta compra, además de los puestos de la compañía pasó a comercializar combustibles en 11 aeropuertos y 7 terminales de distribución, respondiendo por el 16\% del mercado minorista chileno y $7 \%$ del sector industrial (Petrobras 2009).

Con relación a los demás países de América, algunos otros puntos merecen ser resaltados. En Ecuador, desde 1996 Petrobras negocia la exploración de 
petróleo en aguas profundas en la costa del país, en conjunto con otras multinacionales (Bacoccina 2006). En México, desde 2003 estableció acuerdos de cooperación tecnológica en aguas profundas con la empresa estatal Petróleos Mexicanos, Pemex (Petrobras 2007a). Desde 2003 en Venezuela explora y produce petróleo, además de mantener convenios con Petróleos de Venezuela S.A. (PDVSA) para ampliar sus actividades de petróleo y gas en el país (Petrobras 2007a). Y en Paraguay, donde actúa desde 2006, compró negocios de Shell, opera estaciones de servicio por todo el país, y comercializa GLP y productos de aviación (Petrobras 2007a).

Además de eso, actúa también en actividades exploratorias en el mar cubano desde el inicio del año 2000. Petrobras volvió a invertir en Cuba a partir de 2008, por medio de un contrato para trabajar conjuntamente en la exploración y producción de petróleo y gas. Este contrato fue acordado con la Compañía Cubana de Petróleo (CUPET) el 31 de octubre de 2008. El bloque está localizado en aguas profundas (entre 400 y 1.600 metros), en una región próxima a la franja petrolífera cubana continental. El contrato tiene duración de 32 ańos, con un período exploratorio de 7 ańos y 25 años para la producción (Petrobras 2010).

El Cuadro 1 resume la actuación internacional de la empresa en el continente americano. 


\section{Cuadro 1: Distribución espacial y cronológica de inserción de Petrobras en el continente americano, 1972-2007}

\begin{tabular}{|c|c|c|}
\hline País & $\begin{array}{c}\text { Año de } \\
\text { início de las } \\
\text { actividades }\end{array}$ & Actividades \\
\hline Colombia & 1972 & $\begin{array}{l}\text { Exploración de petróleo en aguas rasas y profundas (a partir } \\
\text { de 2004). }\end{array}$ \\
\hline Estados Unidos & 1987 & $\begin{array}{l}\text { Exploración, producción y comercialización de petróleo y } \\
\text { derivados; exploración en aguas profundas a partir de } 2004 \text {. }\end{array}$ \\
\hline Argentina & 1993 & $\begin{array}{c}\text { Exploración, producción y comercialización de petróleo, gas } \\
\text { y derivados. }\end{array}$ \\
\hline Bolivia & 1995 & Exploración, producción y comercialización de gas y petróleo. \\
\hline Ecuador & 1996 & Exploración y producción de petróleo. \\
\hline Venezuela & 2003 & Exploración y producción de petróleo en tierra y offshore. \\
\hline Perú & 2003 & Exploración y producción de petróleo y gas en tierra. \\
\hline México & 2003 & $\begin{array}{l}\text { Exploración y producción de gas natural; acuerdos de } \\
\text { cooperación tecnológica para exploración de petróleo en } \\
\text { aguas profundas. }\end{array}$ \\
\hline Uruguay & 2004 & Comercialización de gas y derivados de petróleo. \\
\hline Chile & 2005 & $\begin{array}{l}\text { Oficina comercial. A partir de 2007, adquisición de red de } \\
\text { puestos de distribución. }\end{array}$ \\
\hline Paraguay & 2006 & Comercialización de combustibles. \\
\hline Cuba & 2008 & Exploración de petróleo en aguas profundas. \\
\hline
\end{tabular}

Fuente: Elaboración de los autores a partir de Petrobras (2007b) y Petrobras (2010b).

Según Costa (2003), "hoy Petrobras es completamente diferente de lo que era una década atrás. Es la segunda mayor empresa de petróleo en Argentina y la primera en Bolivia”. Esta misma visión puede encontrarse en las declaraciones de Planinscheck, Gerente de Relaciones Societarias de Petrobras (2003), cuando afirma que "el principal país es la Argentina, donde están nuestros mayores activos. A pesar del foco estratégico de la empresa ser América Latina, la costa Oeste de África y el Golfo de México, estamos monitoreando todo el mundo. Así y todo, nuestra fortaleza es América Latina”. 
En casi la totalidad de los casos, Petrobras tiene convenios con empresas estatales u otras multinacionales. Como destaca Cretious (2007), Petrobras hizo joint ventures para "tener acceso a la experiencia y conocimiento del mercado internacional, llevando a cabo acuerdos comerciales con socios locales".

\section{Petrobras Internacional en África}

Petrobras tuvo varias experiencias aisladas en África con participación menor en contratos de otras grandes compañías. Pero fue en 1979 que estableció bases sólidas en Angola, donde mantiene contratos de exploración y producción a través de la participación en la extracción de petróleo offshore (Petrobras 2007a). A partir de 2004 cerró contratos con el gobierno angolano para explorar petróleo en aguas profundas, y desde entonces continúa expandiendo sus actividades.

También es importante destacar su ingreso en Nigeria, en 1998, centrado en actividades de exploración y posterior producción en las aguas profundas del delta del río Níger. En 2004 Petrobras consiguió la concesión para explorar campos offshore con más de 2.500 metros de profundidad (Petrobras 2007a).

Su actuación en el continente africano fue significativamente ampliada a comienzos del siglo XXI. En junio de 2004, por ejemplo, inició actividades en Tanzania, cuando firmó contrato con la compañía estatal de petróleo, ampliando su actuación para el este del continente. El acuerdo firmado prevé la exploración en aguas de 500 a 3.500 metros de profundidad (Petrobras 2007a).

En enero de 2005, dio inicio a sus actividades en Libia, cuando ganó una licitación para explorar gas y petróleo en el Mar Mediterráneo en aguas profundas (Petrobras 2007a). En enero de 2006 consiguió la participación en un contrato de exploración conjunta de petróleo en Guinea Ecuatorial, en profundidades variando entre 500 y 2.200 metros (Petrobras 2007a). Finalmente, en octubre de 2006 firmó un memorándum con la Empresa Nacional de Hidrocarburos, de Mozambique, para la exploración de petróleo y gas en tierra y mar. El documento incorpora también la realización de investigaciones y futura producción de biocombustibles en aquel país: biodiesel a partir de la fatrofa, una oleaginosa abundante en la vegetación local, y etanol a partir de la caña de azúcar.

Nuevamente, la casi totalidad de los casos envuelve acuerdos entre Petrobras y empresas estatales $\mathrm{u}$ otras multinacionales. El Cuadro 2 a continuación resume la actuación de la empresa en el continente africano. 


\section{Cuadro 2: Distribución espacial y cronología de inserción de Petrobras en el continente africano, 1979-2007}

\begin{tabular}{ccc} 
País & $\begin{array}{c}\text { Ańo de inicio de las } \\
\text { actividades }\end{array}$ & Actividades \\
\hline Angola & 1979 & $\begin{array}{c}\text { Exploración y producción de petróleo en aguas rasas } \\
\text { y profundas. }\end{array}$ \\
Nigeria & 1998 & $\begin{array}{c}\text { Exploración y producción de petróleo en aguas } \\
\text { profundas. }\end{array}$ \\
Tanzania & 2004 & $\begin{array}{c}\text { Exploración y producción de petróleo en aguas } \\
\text { profundas. }\end{array}$ \\
Libia & 2005 & $\begin{array}{c}\text { Exploración de gas y petróleo en aguas rasas y } \\
\text { profundas. }\end{array}$ \\
Guinea Ecuatorial & 2006 & $\begin{array}{c}\text { Exploración de petróleo en aguas profundas. } \\
\text { Mozambique }\end{array}$ \\
Senegal & 2006 & $\begin{array}{c}\text { Exploración de petróleo y gas natural; acuerdos para } \\
\text { producción local de biocombustibles. }\end{array}$ \\
& 2007 & Exploración de petróleo.
\end{tabular}

Fuente: Elaboración de los autores a partir de Petrobras (2007b) y Petrobras (2010b).

\section{Petrobras Internacional en Eurasia}

En Europa, Petrobras posee desde 1997 una representación comercial y financiera situada en Londres, que busca la consecución de transacciones de compra y venta de petróleo; leasing, alquiler, y otras operaciones relacionadas con equipamientos; además de buscar financiamiento externo (Petrobras 2007a). La empresa no había conseguido obtener espacio para mayores incursiones productivas y comerciales en Europa, un mercado ya ocupado por grandes empresas, principalmente europeas y norteamericanas.

Dos oportunidades, sin embargo, surgieron en 2007. La primera, fruto de la firma de un memorándum con la Statoil, empresa estatal de petróleo de Noruega. El documento prevé, entre otras cosas, la cooperación en investigación y desarrollo de tecnologías para la producción de etanol y biodiesel, así como la cooperación en el desarrollo de proyectos tecnológicos para exploración y producción de petróleo en aguas ultra profundas. La segunda oportunidad, más concreta, tuvo inicio en 2007, a partir de negociaciones celebradas un año antes en Portugal. Así, Petrobras comenzó a explorar petróleo en la costa lusitana, en aguas de hasta 2.000 metros de profundidad. 
En Asia, la firma se instaló primero en Singapur, también con una representación comercial y financiera para tratar, inicialmente, dar sustento a actividades comerciales en el área del petróleo y derivados en la región (Petrobras 2007a). La representación fue importante para que, en el año 2000, la empresa entrara a Japón a través de Petrobras International Finance Company. Con sede en Tokio, la subsidiaria tenía como función principal generar relaciones estrechas con financistas situados en Japón, pero ya desarrolló otras iniciativas. En 2005, el Cenpes firmó acuerdos con la empresa estatal japonesa de petróleo, Jogmec, para estudios tecnológicos conjuntos. En 2007, inició sus primeras actividades de refinamiento de petróleo en Asia y también firmó un nuevo convenio con Jogmec para explorar y producir de manera conjunta petróleo en aguas profundas, tanto en América del Sur como en el Sudeste asiático. A partir de 2008, Petrobras comenzó a vender etanol brasileño en el mercado japonés (Petrobras 2007a).

Otro movimiento importante del Área internacional de Petrobras en Asia fue la firma de un convenio con la empresa estatal china Sinopec en mayo de 2004. Este acuerdo permitió la instalación de una oficina en China, que busca ganar espacio para la venta del petróleo brasileño en ese país, servir de base para otras negociaciones con países asiáticos y ampliar sus actividades con Sinopec. En 2005, el acuerdo entre las empresas fue formalizado, posibilitando el desarrollo de negocios conjuntos, principalmente para exploración y producción de petróleo offshore en las costas de China y del Brasil (Petrobras 2007a).

Vale la pena destacar muchos otros momentos relevantes. Petrobras consiguió en 2004, por ejemplo, ganar una licitación para la exploración de petróleo en el Golfo Pérsico en Irán, una región densamente ocupada por otras grandes empresas. En 2006, pasó a operar en Turquía, ganando la concesión para explorar y producir petróleo en aguas profundas del Mar Negro (Petrobras 2007a). Lo mismo sucedió en Paquistán, en 2007, para explorar y producir petróleo en aguas de hasta 2.000 metros de profundidad. Finalmente, también en 2007, la empresa desarrolló un acuerdo con la empresa estatal de petróleo de la India, para la exploración conjunta de aguas profundas en las costas de ambos países.

De la misma forma que en los casos presentados con anterioridad, todas las actividades citadas envuelven algún tipo de convenio entre Petrobras y empresas estatales u otras multinacionales. Se observa que prácticamente todos los acuerdos en grandes mercados productores de petróleo están asociados a la exploración offshore y, frecuentemente, envuelven áreas de grandes profundidades. Esta característica muestra la superioridad 
tecnológica alcanzada por Petrobras, que es reconocida en el escenario internacional de exploración de petróleo en aguas profundas y, por lo tanto, un importante elemento impulsor de su internacionalización.

El Cuadro 3 presenta un resumen de la actuación internacional de Petrobras en Europa y Asia.

\section{Cuadro 3: Distribución espacial y cronología de inserción de Petrobras en Eurasia, 1979 - 2007}

\begin{tabular}{|c|c|c|}
\hline País & $\begin{array}{l}\text { Año de } \\
\text { inicio de las } \\
\text { actividades }\end{array}$ & Actividades \\
\hline Reino Unido & 1997 & $\begin{array}{l}\text { Representación comercial y financiera; acuerdos para futura } \\
\text { comercialización de biocombustibles. }\end{array}$ \\
\hline Japón & 2000 & $\begin{array}{l}\text { Representación comercial y financiera; refinamiento de petróleo } \\
\text { desde noviembre de 2007; venta de etanol a partir de } 2008 \\
\text { (Orelana y Neto 2006); acuerdo de cooperación tecnológica con la } \\
\text { empresa estatal japonesa Jogmec desde } 2005 \text {. }\end{array}$ \\
\hline Holanda & 2001 & $\begin{array}{c}\text { Representación comercial y financiera. Oficina desactivada } \\
\text { posteriormente. }\end{array}$ \\
\hline China & 2004 & $\begin{array}{c}\text { Representación comercial y financiera, acuerdos comerciales con } \\
\text { Sinopec (empresa estatal china de petróleo) para exploración de } \\
\text { petróleo offshore en China y en Brasil. }\end{array}$ \\
\hline Irán & 2004 & $\begin{array}{l}\text { Oficina de representación; ejecución de contrato de exploración de } \\
\text { petróleo offshore. }\end{array}$ \\
\hline Turquía & 2006 & Exploración de petróleo en aguas profundas. \\
\hline Pakistán & 2007 & Exploración de petróleo en aguas profundas. \\
\hline India & 2007 & Exploración de petróleo y gas en aguas profundas. \\
\hline Portugal & 2007 & $\begin{array}{c}\text { Exploración de petróleo en aguas profundas; acuerdos para futura } \\
\text { comercialización de biocombustibles. }\end{array}$ \\
\hline Noruega & 2007 & Cooperación en investigación y nuevas tecnologías. \\
\hline
\end{tabular}

Fuente: Elaboración de los autores a partir de Petrobras (2007b).

Otra manera de analizar el proceso de internacionalización de la empresa es verificar su actuación en los diferentes países y continentes por tipo de 
actividad desarrollada, como puede observarse en el Cuadro 4.

\section{Cuadro 4: Actividades y países en que actúa Petrobras, 2011}

Actividades Países de actuación

\begin{tabular}{|c|c|}
\hline $\begin{array}{l}\text { Exploración / } \\
\text { Producción }\end{array}$ & $\begin{array}{c}\text { Brasil, Argentina, Uruguay, Bolivia, Perú, Venezuela, } \\
\text { Colombia, México, Estados Unidos, Portugal, Turquía, } \\
\text { Libia, Benin, Nigeria, Gabão, Angola, Namibia, } \\
\text { Tanzania, Australia, Nueva Zelanda (Brasil más } 19 \\
\text { países). }\end{array}$ \\
\hline $\begin{array}{l}\text { Refinamiento / } \\
\text { Petroquímica }\end{array}$ & $\begin{array}{c}\text { Brasil, Argentina, Estados Unidos, Japón (Brasil más } 3 \\
\text { países). }\end{array}$ \\
\hline $\begin{array}{l}\text { Distribución / } \\
\text { Comercialización }\end{array}$ & $\begin{array}{l}\text { Brasil, Argentina, Uruguay, Paraguay, Chile, Colombia, } \\
\text { Curaçao, Holanda (Brasil más } 7 \text { países). }\end{array}$ \\
\hline Gas / Energía & Brasil, Argentina, Uruguay, Bolivia (Brasil más 3 países). \\
\hline Representación & llaterra, China, Singapur, Japón (5 países). \\
\hline
\end{tabular}

Fuente: Elaboración de los autores a partir de Petrobras (2007b) y Petrobras (2010b).

Según el director del área internacional de la Compañía, Nestor Ceveró: "Petrobras no actúa más en el exterior con el objetivo del abastecimiento del mercado brasileño desde 2003. La meta ahora es invertir en la búsqueda de rentabilidad, sin filantropía y con base en contratos" (Marín 2006). El plan estratégico de la empresa refleja esta nueva directriz para la continuidad de su internacionalización. Las inversiones previstas para el mercado externo en el período 2006-2010 eran aproximadamente de US\$ 7.100.000.000, $13 \%$ del total a ser invertido (Petrobras 2004). Más o menos la misma proporción puede ser observada en el análisis de la fuerza de trabajo. A final de 2006, de un total de 62.286 empleados, 6.877 estaban orientados en actividades internacionales (Petrobras 2006). El Plan de Negocios 2008-2012 vislumbraba el objetivo de hacer de Petrobras una de las cinco mayores empresas integradas de energía del mundo (Petrobras 2007b).

\section{CONCLUSIÓN}

A lo largo de séis décadas de existencia, Petrobras se tornó la mayor multinacional brasileña. Su actuación en el exterior reconoce como origen la "búsqueda de recursos naturales" (Dunning 1988) que no estaban disponibles en cantidad suficiente en territorio nacional. Así, fue a Colombia a explorar petróleo en campos onshore. Santos (2003) en su declaración 
recuerda los factores que llevaron a la creación de Braspetro, entre los cuales destaca "la importancia de tener mano de obra internacionalizada, generar acuerdos para el know-how y tecnología con otras empresas, garantizar el abastecimiento de petróleo y derivados para el país", una vez que, al iniciar su internacionalización Petrobras importaba cerca del 80\% del petróleo consumido en Brasil.

Desde la década de 1990 otras fuerzas ganaron importancia en este movimiento. La mayor internacionalización de la empresa a partir de aquel momento fue parte de la estrategia de conquistar nuevos mercados y tornarse líder en América Latina. Para cumplir con su propósito original, la firma fue capaz de hacer un catching up tecnológico, tornándose referencia mundial en tecnologías offshore (Ortiz Neto 2006).

Su competencia en el área es hoy reconocida mundialmente, algo que le permite materializar contratos para exploración y producción alrededor del mundo en aguas profundas y ultra profundas todavía poco exploradas. Además de eso, tal competencia ha sido requerida para asociaciones con otras gigantes del sector para la ejecución de contratos de exploración y para trabajos conjuntos de investigación y desarrollo tecnológico (JB 2007). Proyectando la autosuficiencia para el inicio del nuevo milenio, la empresa reformuló sus estrategias para tratar de crecer internacionalmente más allá de América Latina (Cretious 2007). O sea, su nueva estrategia de internacionalización se dio a partir de la construcción de "propiedades intangibles" (Dunning 1988). Además de eso, la empresa se ha encaminado en una estrategia osada para tratar de crear mercados para biocombustibles y así aprovechar comercialmente el conocimiento y las tecnologías propias que desarrolló a lo largo de su historia.

De a poco, fue consolidando su experiencia internacional que contribuyó para colocarla como uno de los principales jugadores mundiales en el negocio del petróleo. Este hecho puede ser percibido tanto por el alcance geográfico de su actuación (América, Eurasia y África) como por la disputa en la adquisición de nuevos bloques exploratorios en la venta de tecnología offshore, en los joint ventures firmados con otras multinacionales y, finalmente, por la mano de obra altamente calificada y con expresiva experiencia internacional.

Si nos basamos en los "niveles de internacionalización" descritos por Johanson y Wiedersheim-Paul (1999) podemos percibir que Petrobras comenzó de manera tímida e incipiente en un ambiente ya dominado por grandes multinacionales para, de a poco, transformarse en una multinacional de competencia en la vanguardia tecnológica actuando en 26 países y facturando en 2006 el 15\% de sus ingresos en el mercado 
externo. En una época en la cual se habla mucho de la intensidad de la globalización, la historia de internacionalización de Petrobras es ejemplo de la competencia brasileña no sólo en producir commodities y bienes intensivos en mano de obra, sino también en construir ventajas competitivas y vanguardia tecnológica.

Estas ventajas en la vanguardia tecnológica son también responsables por uno de los grandes descubrimientos mundiales de petróleo en los últimos años, que es el pre- sal. Petrobras, a través de la tecnología offshore puede no sólo descubrir este petróleo y gas, sino también dar inicio al proceso de exploración, aunque la tecnología para esto no este del todo desarrollada. 


\section{REFERENCIAS}

Alem, A. C. y Cavalcanti, C. E. (2005). O BNDES e o Apoio à Internacionalização das Empresas Brasileiras: Algumas Reflexôes. Revista do BNDES, 2 (4), 43-76.

Alveal, C. (2008). A Petrobrás na Economia Global: Desafios e Oportunidades de uma Estatal de Trajetória Singular. En Dalla Costa, A., Fernandes, A. S. y Szmrecsányi, T. (Eds.), Empresas, Empresários e Desenvolvimento Econômico no Brasil. São Paulo: Hucitec/ABPHE.

Agência Nacional do Petróleo, Gás Natural e Biocombustível (ANP). (2007). Resumo das Rodadas Realizadas. Disponible en http://www. brasil-rounds.gov.br/geral/resumo_geral_R9.asp\# [06-12-2007].

Bacoccina, D. (2006). Lula Promete Investimento Brasileiro no Equador. Disponible en http://www.bbc.co.uk/portuguese/reporterbbc/ story/2007/04/070404_rafaelcorreadb_ac.shtml [10-12-2007].

Buckley, P. y Casson, M. (1976). The Future of the Multinational Enterprise. Londres: Macmillan.

Buckley, P. y Casson, C. (1981). The Optimal Timing of a Forein Direct Investment. Economic Journal, 91, 75-8.

Caves, R. E. (1980). Multinational Entreprise and Economic Analysis. Cambridge: Cambridge University Press.

Chandler, A. D. (1962). Strategy and Structure. Cambridge: The MIT Press.

. (1977). The Visible Hand: The Managerial Revolution in American Business. Cambridge: Harvard University Press.

Costa, D. (2003). Depoimento para o Projeto Memória dos Trabalhadores Petrobras. Disponible en http://memoria.petrobras.com.br/internauta/ conteudo/ifrmDepoimentoCompleto.jsp [15-02-2007].

Cretious, S. (2007). Internacionalização de Pequenas e Médias Empresas. En Almeida, A. (Ed.), Internacionalizaçẫo de Empresas Brasileiras. Perspectivas e Riscos. Rio de Janeiro: Elsevier.

Dunning, J. H. (1988). Explaining International Production. Londres: Unwin Hyman. 
(2001). The Eclectic Paradigm of International Production: A Personal Perspective. En Pitelis, C. y Sugden, R. (Eds.), The Nature of Transnational Firm. Londres: Routledge.

G1. (2013). Petrobras Tem Lucro Líquido de R\$ 21,18 Bilhóes em 2012, Queda de 36\%. Disponible en http://g1.globo.com/economia/ negocios/noticia/2013/02/petrobras-tem-lucro-liquido-de-r-2118bilhoes-em-2012-queda-de-36.html [08-02-2013].

Hymer, S. (1968). The Large Multinational Corporation. En Casson, M. (Ed.), Multinational Corporation. Hants: Edward Elgar.

Ipedata. (2009). Importação, Produção e Consumo Aparente de Petróleo. Disponible en http://www.ipeadata.gov.br, 2009 [01-02-2013].

Johanson, J. y Wiedershein-Paul, F. (1999). The Internationalization of the Firm: Four Swedish Cases. En Buckley, P. J. y GhaurI, P. N. (Eds.), The Internationalization of the Firm. Londres: International Thomson Business Press.

Journal do Brasil (JB). (2007). Estatal Firma Acordo Tecnológico com StatoilHydro. Journal do Brasil, 4 de diciembre de 2007.

Lall, S. (1983). Technological Change, Employment Generation and Multinationals: a Case Study of a Foreign Firm and a a Local Multinational in India. Ginebra: OIT.

Lecraw, D. (1977). Direct Investment by Firms from Less Developed Countries. Oxford Economic Papers, 29, 442-457.

Marin, D. (2006). Política Externa Ajuda Petrobrás. O Estado de São Paulo, 19 de febrero de 2006.

Mckain, D. y Allen, B. (1994). Where it All Began the Story of the People and Places where the Oil \& Gas Industry Began. Parkersburg: Oil \& Gas Museum.

Moura, M. (Ed.). (2003). Petrobras 50 Anos. Rio de Janeiro: Petrobras.

Ortiz Neto, J. B. (2006). O Processo de Aprendizado Tecnológico na Trajetória do Sistema de Produção Flutuante Empreendido pela Petrobrás em seu Programa de Capacitação Tecnológica em Águas Profundas - PROCAP. Tesis de Maestría, Programa de Pós-Graduação em Desenvolvimento Econômico, Universidade Federal do Paraná, Brasil. 
Pitelis, N. y Sugden, R. (2000). The Nature of the Transnational Firm. Nueva York: Routledge.

Petrobras. (2003). Relatório Anual. Disponible en http://www. petrobras. com.br [01-05-2013].

. (2004) Relatório Anual. Disponible en http://www. petrobras.com. br [01-05-2013].

. (2006). Efetivo de Pessoal - Sistema Petrobras 2006. Disponible en http://www2.petrobras.com.br/ri/port/ConhecaPetrobras/ RecursosHumanos/pdf/EfetivoPessoal_2006_Port.pdf [12-02-2006].

. (2007a). Atuação Internacional. Disponible en http://www2. petrobras.com.br/portal/AtuacaoInternacional.htm. Acesso em: [1212-2007].

. (2007b). Plano Estratégico Petrobras 2008-2012. Disponible en http://www2.petrobras.com.br/portugues/ads/ads_Petrobras.html [17-12-2007].

. (2008). Relatório Anual. Disponible en http://www. petrobras.com. br [01-05-2013].

- (2009a). Petrobras em Números. Disponible en http://www2. petrobras.com.br/ portugues/ads/ads_Petrobras.html [31-07-2009].

- (2009b). PROCAP 3000: Próxima Fronteira de Exploração em Águas Ultraprofundas. Disponible en http://www2.petrobras. com.br/portal/ frame.asp?pagina=/tecnologia/port/centro_ pesquisasdapetrobrasexpansao.asp\&lang=pt\&area=tecnologia [19-082009].

. (2009c). Relatório Anual - 2008. Disponible en http://www2. petrobras.com.br [20-08-2009].

. (2010). Petrobras no Mundo. Disponible en http://www.petrobras. $\mathrm{com} / \mathrm{ptcm} /$ appmanager/ptcm/dptcm?_nfpb=true\&_pageLabel=petr_ com_mundo [31-01-2011].

. (2011). Relatório Anual. Disponible en http://www. petrobras.com. br [01-05-2013].

. (2012). Relatório Anual. Disponible en http://www. petrobras.com. br [01-05-2013]. 
. (2013). Relatório Anual. Disponible en http://www. petrobras.com. br [01-05-2013].

Santos, S. P. (2003). Depoimento Captado em 2003 para Registrar a Memória dos Trabalhadores da Petrobras. Brasilia: Petrobras.

Sciarretta, T. (2010). Petrobras Capta R \$ 120,4 bi com Ações. Folha de São Paulo, 23 de septiembre. Disponible en http://www1.folha.uol. com.br/mercado/803932-petrobras-capta-r-1204-bi-com-acoes.shtml [01-05-

United Nations Conference on Trade and Development (UNCTAD) (2007). World Investment Report. Transnational Corporations, Extractive Industries and Development. Nueva York: Naciones Unidas.

\section{Periódicos}

Folha de Sao Paulo, 2012.

Recibido: 30-11-2012

Aceptación de la versión final: 10-04-2013 